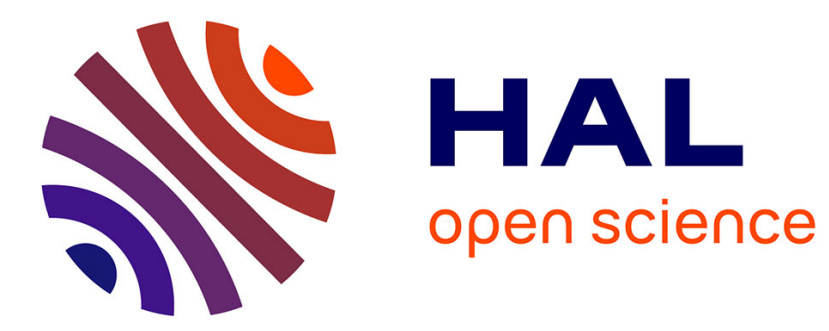

\title{
How to extract information from simulations of coarsening at finite temperature
}

Bernard Derrida

\section{To cite this version:}

Bernard Derrida. How to extract information from simulations of coarsening at finite temperature. Physical Review E , 1997, 55 (3), pp.3705-3707. 10.1103/PhysRevE.55.3705 . hal-03282971

\section{HAL Id: hal-03282971 \\ https://hal.science/hal-03282971}

Submitted on 19 Jul 2021

HAL is a multi-disciplinary open access archive for the deposit and dissemination of scientific research documents, whether they are published or not. The documents may come from teaching and research institutions in France or abroad, or from public or private research centers.
L'archive ouverte pluridisciplinaire HAL, est destinée au dépôt et à la diffusion de documents scientifiques de niveau recherche, publiés ou non, émanant des établissements d'enseignement et de recherche français ou étrangers, des laboratoires publics ou privés. 


\title{
How to extract information from simulations of coarsening at finite temperature
}

\author{
B. Derrida \\ Laboratoire de Physique Statistique, ENS, 24 rue Lhomond, 75005 Paris, France \\ and Service de Physique Théorique, CE Saclay, F91191 Gif-sur-Yvette, France
}

(Received 28 October 1996)

\begin{abstract}
Certain properties of coarsening phenomena such as the fraction of space which remains always in the same phase are hard to measure in simulations, at nonzero temperature, because one needs to distinguish spin flips due to thermal fluctuations from those due to the motion of interfaces. By comparing the system where coarsening takes place with a fully ordered system submitted to the same thermal noise, one can measure in a simple way the fraction of space which remains in the same phase. Our numerical results indicate a power law decay (of the fraction of persistent spins) in the whole low temperature phase very similar to the zero temperature situation. [S1063-651X(97)01503-1]

PACS number(s): 02.50.- r, 05.40. $+\mathrm{j}, 05.20 .-\mathrm{y}$
\end{abstract}

Ising or Potts models evolving according to Glauber dynamics are simple examples of systems exhibiting coarsening $[1,2]$. Apart from the one dimensional case, where coarsening takes place only at zero temperature [3-6], very few properties are known analytically and the only possible approach (to test approximate methods) is based on numerical simulations [1]. As soon as the temperature is nonzero, some properties have to be defined in a careful way in order to be extracted from simulations. The goal of this paper is to propose a possible method of measuring properties related to coarsening in the presence of thermal fluctuations.

In dimension higher than 1 , below the ordering temperature (which is nonzero), if one starts with a fully random configuration, Glauber dynamics tends to align neighboring spins and to produce a pattern of ferromagnetic domains with a typical domain size which grows with time as $t^{1 / 2}$. In simulations, one can measure the pair correlation between two spins a distance $R$ apart and check [1] that indeed in the long time limit (and for $R$ large compared to the bulk correlation length at equilibrium), it becomes a scaling function of the ratio $R / \sqrt{t}$. A number of difficulties arise if one tries to measure more complicated properties such as the distribution of domain sizes or the number of persistent spins [5,7-12,22].

At zero temperature, one can easily define domains as connected sets of parallel spins and the fraction of persistent spins as the fraction of spins which never flip up to time $t$. A serious difficulty, however, is that at zero temperature, the dynamics can be greatly affected by local blocking configurations $[13-15,9]$ of the interface (finite barriers of energy can stop the dynamics) and so one may wonder whether the absence of thermal fluctuations allowing one to overcome these finite energy barriers could not make the zero temperature coarsening a separate problem which behaves in a completely different way than coarsening at nonzero temperature.

At nonzero temperature, it is clear that finite energy barriers cannot stop the dynamics. However, it becomes difficult to define domains because it is hard to distinguish between true domains and spin flips only due to thermal fluctuations. Also at any nonzero temperature, each spin, even if it points in the direction of its local field, has always a nonzero probability per unit time of flipping and the number of spins which do not flip up to time $t$ decreases exponentially with time. However, it is clear that when the typical domain size becomes much larger than the equilibrium bulk correlation length, it is possible to tell which domain is in which phase and one can then ask what is the fraction of space which remains in the same phase up to time $t$. The goal of the present paper is to give a way of measuring this fraction of space even in the presence of thermal fluctuations.

The main idea developed here consists in comparing the system (system $A$ ) where coarsening takes place with an ordered system (system $B$ ) when both systems are submitted to the same noise $[16,17,23]$. Then, when a spin flips in system $A$, if the same spin flips in system $B$, one can consider that this is due to thermal fluctuations whereas when a spin behaves differently in systems $A$ and $B$, one considers that this is due to the motion of the domain walls in the coarsening process.

In practice, in the case of an Ising model at temperature $T$, the two systems $A$ and $B$ of $N$ spins are identical except for their initial condition

$$
S_{i}^{A}(0)=\left\{\begin{array}{lll}
+1 & \text { with probability } & 1 / 2 \\
-1 & \text { with probability } & 1 / 2
\end{array}\right.
$$

and

$$
S_{i}^{B}(0)=+1
$$

and Glauber dynamics is performed by updating in both systems during each time interval $\Delta t=1 / N$ the same randomly chosen spin $i$ according to

$$
\begin{aligned}
& S_{i}^{A}(t+\Delta t)=\operatorname{sgn}\left[1+\tanh \left(\frac{\sum_{j} S_{j}^{A}(t)}{T}\right)-2 z(t)\right], \\
& S_{i}^{B}(t+\Delta t)=\operatorname{sgn}\left[1+\tanh \left(\frac{\sum_{j} S_{j}^{B}(t)}{T}\right)-2 z(t)\right],
\end{aligned}
$$

where in Eqs. (3) and (4) the sums over $j$ run over all the neighbors of site $i$ and the same random number $z(t)$, uniformly distributed between 0 and 1 , is used.

To measure the fraction $r(t)$ of persistent spins, one just calculates 


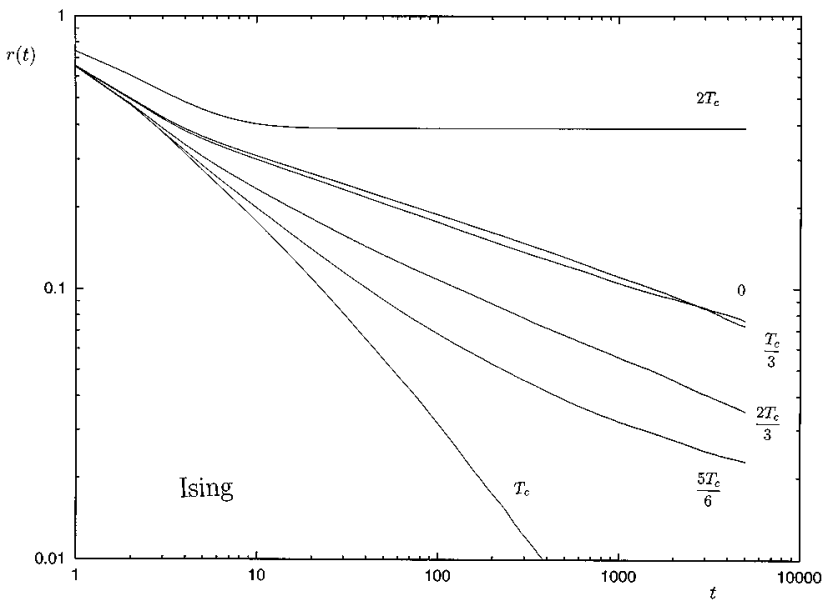

FIG. 1. Fraction $r(t)$ of spins remaining identical in systems $A$ and $B$ versus time ( $t$ is the number of Monte Carlo steps per spin) for the square lattice with nearest neighbor interactions and periodic boundary conditions. The system size is $L=1000$. Below $T_{c}$, one observes a power law decay.

$$
r(t)=\frac{1}{N} \sum_{i=1}^{N} \prod_{0 \leqslant t^{\prime} \leqslant t} \frac{1+S_{i}^{A}\left(t^{\prime}\right) S_{i}^{B}\left(t^{\prime}\right)}{2}
$$

The effect of the dynamics (3) and (4) is to make the spins of $A$ and $B$ become identical in ferromagnetic regions with positive magnetization. Therefore $r(t)$ measures the fraction of persistent spins in the + phase. For symmetry reasons between positive and negative magnetizations, it is clear that for a large enough system, the fraction of persistent spins should be $2 r(t)$.

Figure 1 shows a log-log plot of $r(t)$ measured for an Ising model on a square lattice of $1000 \times 1000$ spins at several temperatures (for a single sample). Below $T_{c}, r(t)$ decays as power law

$$
r(t) \sim t^{-\theta}
$$

with an exponent $\theta$ close to its zero temperature value. From Fig. 1, one can estimate

$$
\theta \simeq \begin{cases}0.22 & \text { for } T=0 \\ 0.22 & \text { for } T=T_{c} / 3 \\ 0.29 & \text { for } T=2 T_{c} / 3 .\end{cases}
$$

Right at $T_{c}$, the decay is much faster than in the low temperature phase and the fraction of persistent spins [as defined by Eq. (5)] becomes very small so quickly that it is hard to analyze the type of decay. As $T$ approaches $T_{c}$ from below (case $T=5 T_{c} / 6$ ), one observes a crossover between the power law characteristic of the low temperature phase and the rapid decay at $T_{c}$. Above $T_{c}$, when two systems are submitted to the same noise, they become identical very quickly [16] and therefore $r(t)$ does not decay to zero as $t \rightarrow \infty$. This result looks somewhat strange if one thinks of the persistent spins as spins which keep the same magnetization up to time $t$. On the other hand, if one thinks of the persistent spins as being the spins which remain in the same phase up to time $t$, it is clear that above $T_{c}$, there is a single phase (the disordered phase) and so it is not hard to accept that the fraction of space remaining in the same phase remains nonzero in the long time limit.
Figure 1 shows rather convincingly that $r(t)$ has a power law decay in the whole low temperature phase. A natural question, of course, is that of universality, namely, whether the exponent $\theta$ is independent of temperature. From the estimates of Eq. (7), $\theta$ does not seem to change much with temperature, and so one could attribute its apparent variation to a lack of accuracy of the estimates (7) and to an insufficient numerical effort. On the other hand, it has been shown recently [18] that anisotropy due to the lattice affects coarsening even in its long time regime, at least through the angular dependence of the surface tension of an interface at equilibrium. As this angular dependence depends on temperature, one can expect that changing the temperature may change the whole universality class of the coarsening phenomena and this would imply that $\theta$ does vary with temperature. At present, it seems that numerical simulations, at least one order of magnitude more than what is presented here, would be necessary to distinguish clearly (by measuring $\theta$ ) between these two possibilities.

The above way of measuring the fraction of persistent spins can be extended to the case of the $q$-state Potts model: for system $A$, each spin $S_{i}$ is given initially one out of the $q$ possible colors at random whereas for system $B$, each spin is initially in color 1 . Then to implement the heat bath dynamics, one chooses at each time step $\Delta t=1 / N$, a site $i$ at random among the $N$ spins of the system and a random number $z(t)$ uniformly distributed between 0 and 1 . One calculates for each system the probabilities $P^{A}(\alpha)$ and $P^{B}(\alpha)$ that spin $S_{i}$ takes the color $\alpha$ at time $t+\Delta t$,

$$
\begin{aligned}
& P^{A}(\alpha)=\exp \left(\frac{1}{T} \sum_{j} \delta_{S_{j}^{A}(t), \alpha}\right)\left[\sum_{\gamma=1}^{q} \exp \left(\frac{1}{T} \sum_{j} \delta_{S_{j}^{A}(t), \gamma}\right)\right]^{-1}, \\
& P^{B}(\alpha)=\exp \left(\frac{1}{T} \sum_{j} \delta_{S_{j}^{B}(t), \alpha}\right)\left[\sum_{\gamma=1}^{q} \exp \left(\frac{1}{T} \sum_{j} \delta_{S_{j}^{B}(t), \gamma}\right)\right]^{-1},
\end{aligned}
$$

where the sums over $j$ run over all the neighbors of the site $i$ and one updates the two systems according to

$$
\begin{aligned}
& S_{i}^{A}(t+\Delta t)=\alpha_{A}, \\
& S_{i}^{B}(t+\Delta t)=\alpha_{B},
\end{aligned}
$$

where the colors $\alpha_{A}$ and $\alpha_{B}$ are determined by

$$
\begin{aligned}
& \sum_{\gamma=1}^{\alpha_{A}-1} P^{A}(\gamma)<z(t)<\sum_{\gamma=1}^{\alpha_{A}} P^{A}(\gamma), \\
& \sum_{\gamma=1}^{\alpha_{B}-1} P^{B}(\gamma)<z(t)<\sum_{\gamma=1}^{\alpha_{B}} P^{B}(\gamma) .
\end{aligned}
$$

One then measures the fraction $r(t)$ of spins which remain in the phase $\alpha=1$ up to time $t$ as

$$
r(t)=\frac{1}{N} \sum_{i=1}^{N}\left\{\prod_{0 \leqslant t^{\prime} \leqslant t} \delta_{S_{i}^{A}\left(t^{\prime}\right), S_{i}^{B}\left(t^{\prime}\right)}\right\} .
$$

As in the Ising case, the effect of the dynamics (8)-(13) is to make the spins of $A$ and $B$ become identical in regions of 


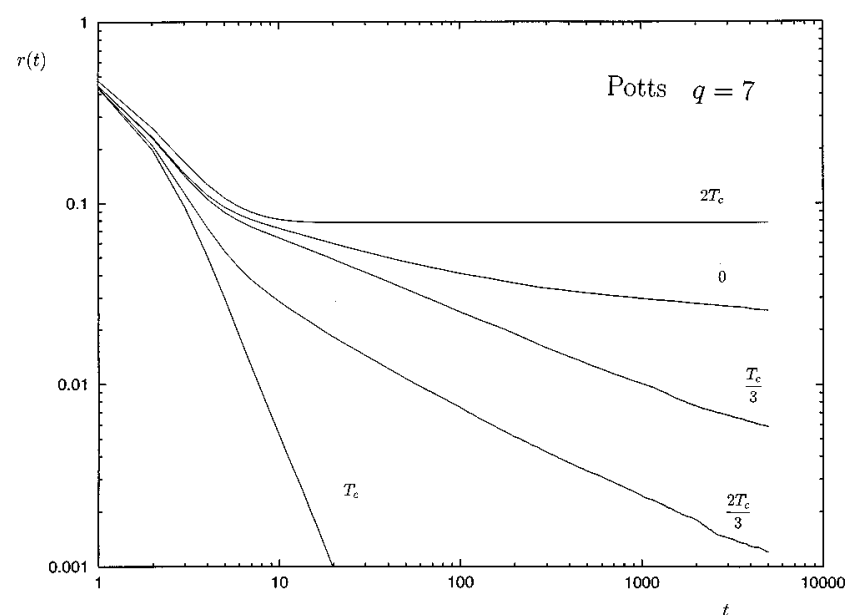

FIG. 2. The same as Fig. 1 for the $q=7$ Potts $\stackrel{t}{\text { model. }}$

phase 1 . Therefore $r(t)$ measures the fraction of persistent spins in phase 1 and for symmetry reasons between the $q$ possible phases, the fraction of spins which remain in the same phase up to time $t$ should be given by $q r(t)$.

At zero temperature, the dynamics of system $B$ is trivial as all the spins of system $B$ remain in color 1 forever and the definition (14) reduces clearly to the fraction of spins of color 1 which never flip. As in the Ising case, at any nonzero temperature, the fraction of spins which never flip would decay exponentially but with definition (14) one finds a power law decay as shown in Fig. 2 for a system of $1000 \times 1000$ on a square lattice for $q=7$. At zero temperature, the decay is very slow because of blocking effects due to finite energy barriers [13-15,9]. These blocking effects are a real problem in zero temperature simulations of the Potts model and so far the only way to overcome them is to take a different lattice (triangular) or to include further neighbor interactions without knowing whether all the undesirable effects due to finite energy barriers have been re- moved. At finite temperature, Fig. 2 shows, however, that $r(t)$ defined by Eq. (14) does decay as a power law with an exponent which seems to vary with temperature (for $q=7$, one can estimate $\theta \simeq 0.40$ at $T=T_{c} / 3$ and $\theta \simeq 0.55$ at $\left.T=2 T_{c} / 3\right)$. As in the Ising case, more numerical work would be needed to confirm that the variation of $\theta$ with temperature is real (and due to the variation of the angular dependence of the surface tension with temperature [18]).

In this paper we have seen that, by using the same thermal noise on the configuration where coarsening takes place and on a fully ordered configuration, one can measure the fraction of spins which remain in the same phase up to time $t$. Very much like at zero temperature, this fraction $r(t)$ has a power law decay in the low temperature phase. Our estimates indicate some variation of the exponent $\theta$ with temperature, but better numerical simulations would be needed to confirm this variation.

The approach used in this paper allows one to define domains at finite temperature. It could therefore be used to measure statistical properties of domains such as the distribution of the sizes of domains. One could also try to extend this approach to measure directly other properties of coarsening at finite temperature, like the characteristic length of the domains: by comparing the system where coarsening takes place (system $A$ ) with a fully ordered system (system $B$ ) submitted to the same noise, one could measure the excess of energy in system $A$ and measure that way how the perimeter of the domains decays with time.

An important case which seems at the moment out of reach with the present approach is the $q=\infty$ Potts model at finite temperature which is of interest in particular in the study of soap froth and for which the value of $\theta$ is still controversial $[9,19-21]$. With the definition (14) one could measure the fraction of space remaining in phase 1 up to time $t$ but this fraction would be vanishingly small in the limit $q \rightarrow \infty$.
[1] A.J. Bray, Adv. Phys. 43, 357 (1994).

[2] P.C. Hohenberg and B.I. Halperin, Rev. Mod. Phys. 49, 435 (1977).

[3] A.J. Bray, J. Phys. A 23, L67 (1990).

[4] J.G. Amar and F. Family, Phys. Rev. A 41, 3258 (1990).

[5] B. Derrida, V. Hakim, and V. Pasquier, Phys. Rev. Lett. 75, 751 (1995).

[6] B. Derrida and R. Zeitak, Phys. Rev. E 54, 2513 (1996).

[7] B. Derrida, A.J. Bray, and C. Godrèche, J. Phys. A 27, L357 (1994).

[8] D. Stauffer, J. Phys. A 27, 5029 (1994); D. Stauffer and R. Knecht, Int. J. Mod. Phys. C 8, 893 (1996).

[9] B. Derrida, P.M.C. de Oliveira, and D. Stauffer, Physica A 224, 604 (1996).

[10] P.L. Krapivsky, E. Ben-Naim, and S. Redner, Phys. Rev. E 50, 2474 (1994).

[11] P.L. Krapivsky, S. Redner, and F. Leyvraz, Phys. Rev. E 51, 3977 (1995).

[12] E. Ben-Naim, L. Frachebourg, and P.L. Krapivsky, Phys. Rev. E 53, 3078 (1996); Phys. Rev. Lett. 77, 2125 (1996); Phys. Rev. E 54, 6186 (1996).
[13] P.S. Sahni, D.J. Srolovitz, G.S. Grest, M.P. Anderson, and S.A. Safran, Phys. Rev. B 28, 2705 (1983).

[14] G.S. Grest, D.J. Srolovitz, and M.P. Anderson, Phys. Rev. B 38, 4752 (1988).

[15] M. Lau, C. Dasgupta, and O.T. Valls, Phys. Rev. B 38, 9024 (1988).

[16] B. Derrida and G. Weisbuch, Europhys. Lett. 4, 657 (1987).

[17] A. Coniglio and L. de Arcangelis, Europhys. Lett. 7, 113 (1988).

[18] A.D. Rutenberg, Phys. Rev. E 54, 2181 (1996).

[19] B. Levitan and E. Domany (unpublished).

[20] W.Y. Tam, R. Zeitak, K.Y. Szeto, and J. Stavans (unpublished).

[21] S. N. Majumdar and C. Sire, Phys. Rev. Lett. 77, 1420 (1996); and unpublished.

[22] S. N. Majumdar, A. J. Bray, S. Cornell, and C. Sire, Phys. Rev. Lett. 85, 763 (1996).

[23] J. S. C. Glotzer, P. H. Poole, and N. Jan, J. Stat. Phys. 68, 895 (1992). 